\title{
乌ூ \\ Subnanosecond spectral diffusion of a single quantum dot in a nanowire
}

\author{
G. Sallen, ${ }^{1}$ A. Tribu, ${ }^{2}$ T. Aichele, ${ }^{3}$ R. André, ${ }^{1}$ L. Besombes, ${ }^{1}$ C. Bougerol,${ }^{1}$ M. Richard,${ }^{1}$ S. Tatarenko, ${ }^{1}$ \\ K. Kheng, ${ }^{2}$ and J.-Ph. Poizat ${ }^{1}$ \\ ${ }^{1}$ CEA-CNRS-UJF Group "Nanophysique et Semiconducteurs," Institut Néel, CNRS, Université Joseph Fourier, F-38042 Grenoble, France \\ ${ }^{2}$ CEA-CNRS-UJF Group "Nanophysique et Semiconducteurs,” CEA/INAC/SP2M, F-38054 Grenoble, France \\ ${ }^{3}$ Physics Institute, Humboldt University, Berlin, Germany
}

(Received 4 May 2011; published 18 July 2011)

\begin{abstract}
We have studied spectral diffusion of the photoluminescence of a single CdSe quantum dot inserted in a $\mathrm{ZnSe}$ nanowire. We have measured the characteristic diffusion time as a function of pumping power and temperature using a recently developed technique [G. Sallen et al., Nat. Photon. 4, 696 (2010)] that offers subnanosecond resolution. These data are consistent with a model where only a single carrier wanders around in traps located in the vicinity of the quantum dot.
\end{abstract}

DOI: 10.1103/PhysRevB.84.041405

PACS number(s): 78.67.Hc, 78.67.Uh, 78.55.Et, 42.50.Lc

Elementary light emitters made of a single quantum object are very sensitive to their local environment. Their optical properties, such as intensity, polarization, and spectrum, can be modified by charge or spin changes in their environment. Spectral diffusion (SD) of a single-light emitter corresponds to random spectral jumps of a narrow line within a broader spectral profile. This effect has been observed in single-molecule ${ }^{1,2}$ or single-semiconductor quantum- $\operatorname{dot}^{3-9}$ experiments. It is generally due to the Stark effect caused by the electric field of randomly trapped charges ${ }^{4-8}$ or to spin fluctuations ${ }^{9}$ in the vicinity of the emitter.

Single-light emitters are very promising objects for quantum information as single photon sources, for flying-solid qubit interface, ${ }^{10}$ or for single-photon manipulation. ${ }^{11-13}$ In quantum cryptography, ${ }^{14,15}$ the spectrum of the single photons can be rather broad, ${ }^{14}$ and SD does not significantly affect the performance. On the contrary, quantum logic operation using linear optics as proposed by Knill et al. ${ }^{16}$ or quantum logic gates using nonlinear interaction at the single-photon level (see Ref. 13 and reference therein) requires indistinguishable single photons with ideal spectral purity. This means that they must necessarily be SD free, which is also the case for quantum memories. A way to be SD free is to operate faster than spectral diffusion. The knowledge of the characteristic time of spectral diffusion is thus required to know whether such a solution is possible.

Visualizing directly the spectral wandering by recording a time series of spectra has been so far the usual method to observe SD. ${ }^{1,3-7}$ For single-photon emitters, the time resolution was therefore limited by the minimum time of about $1 \mathrm{~ms}$ required for a photon-counting charge-coupled device (CCD) to acquire a spectrum. Palinginis et al. ${ }^{17}$ have improved this resolution by measuring a modulation frequencydependent linewidth in a spectral hole-burning experiment using inhomogeneously broadened ensembles of semiconducting nanocrystals. Our recently developed technique ${ }^{18}$ converts spectral fluctuations into intensity fluctuations, as also reported in Refs. 2 and 19-21. It benefits from the subnanosecond time resolution of a Hanbury-Brown and Twiss photon correlation setup and improves by more than 4 orders of magnitude the accessible SD times. It gives access to SD time in the nanosecond range, as shown in this Rapid Communication.
This technique is presented in detail in Ref 18. A sketch of the experimental setup is shown in Fig. 1. In short, it is based on correlations of photons emitted within a spectral window narrower than the SD broadened line. Owing to the wandering of the homogeneous line, the emission energy stays a limited time within this spectral window, leading to photon bunching. The characteristic time of this effect can be easily accessed by photon correlation. Figure 2(a) shows a typical result for autocorrelation on one half of the line, and Fig. 2(b) shows a result for cross correlation between the two halves of the line.

In the present work we use this method ${ }^{18}$ to evaluate the $\mathrm{SD}$ characteristic time of CdSe quantum dots embedded in a $\mathrm{ZnSe}$ nanowire ${ }^{22-24}$ and obtain information about the SD mechanism in this system.

Semiconductor nanowires (NWs) appear to be promising building blocks for nanoscale devices and circuits since they can be grown almost defect free on low-cost, routinely used substrates such as silicon. ${ }^{25}$ Furthermore, NW heterostructures are much less limited by lattice mismatches, which greatly widens the possible material combinations compared to standard self-assembled quantum dots (QDs). Details on the growth of the CdSe/ZnSe NWs can be found in Ref. 22. Their diameter is around $10 \mathrm{~nm}$. An image of the sample is shown in Fig. 3 together with a typical photoluminescence spectrum. Exciton (X), biexciton (XX), and charged exciton (CX) lines have been identified unambiguously using photon-correlation spectroscopy. ${ }^{23}$ According to previously published results, ${ }^{26,27}$ it is commonly admitted that charged excitons in $\mathrm{CdSe}-\mathrm{ZnSe}$ QDs are negatively charged. The radiative lifetimes of these transitions are, respectively, $\tau_{X}=700 \mathrm{ps}, \tau_{X X}=400 \mathrm{ps}$, and $\tau_{C X}=600 \mathrm{ps}$. The luminescence wavelength is around 550 nm with a high count rate of 25000 counts per second at $T=$ $4 \mathrm{~K}$. This system has demonstrated single-photon generation up to a temperature of $T=220 \mathrm{~K}^{24}$ The QD is either neutral (X and XX lines) or charged (CX line). It can be seen in Fig. 3(b) that the CX line is more intense than the $X$ and $X X$ lines, indicating that the QD spends more time in the charged state than in the neutral states. ${ }^{23}$

The SD rate is extracted directly from the time width of the correlation measurement, as shown in Fig. 2. In practice, a more accurate value of the diffusion rate $\gamma_{d}$ is obtained by 


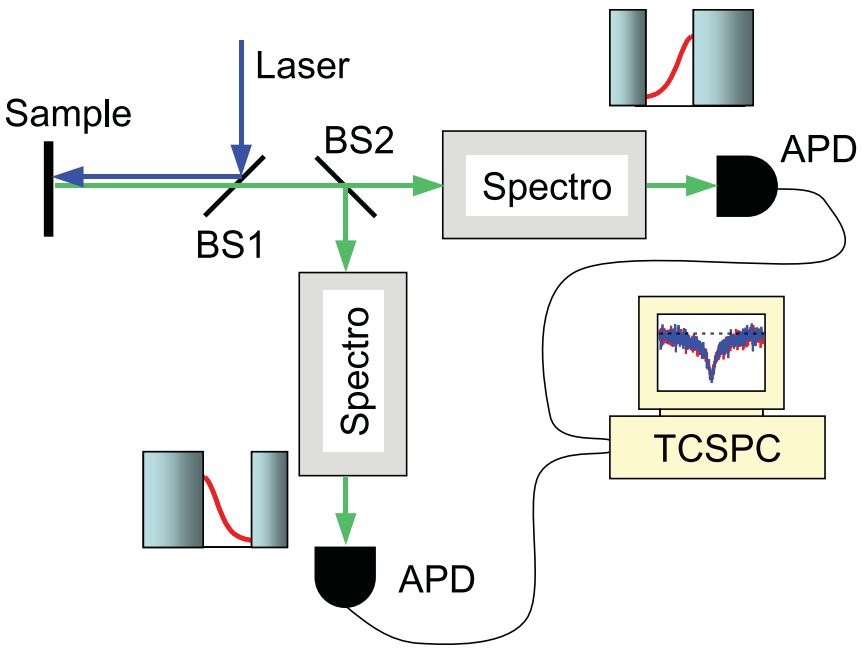

FIG. 1. (Color online) The experimental setup is based on a standard microphotoluminescence experiment operating at a temperature of $T=4 \mathrm{~K}$. A continuous-wave diode laser emitting at $405 \mathrm{~nm}$ excites the sample via beam splitter BS1 with reflection $\mathrm{R} 1=30 \%$. The luminescence is then transmitted through BS1 and split by 50/50 beam splitter BS2. Each beam is sent to a monochromator (resolution of $0.2 \mathrm{meV}$ ) whose output slit illuminates an avalanche photodiode (APD) connected to a time-correlated single-photon (TCSP) module that builds a histogram of the time delays between photons. This allows us to perform either autocorrelation when the two spectrometers (Spectro) are tuned at the same wavelength or a cross correlation otherwise. The output slits of the spectrometers are adjusted so that a controlled spectral window of a given line is detected. The work presented herein has been obtained with high-quantum-efficiency APDs $(\eta=60 \%$ at $550 \mathrm{~nm})$. With these APDs the measured timing resolution of the whole setup is $800 \mathrm{ps}$.

fitting the antibunching trace coming from cross-correlation data [Fig. 2(b)] rather than the fitting from the autocorrelation data [Fig. 2(a)] since the latter contains the excitonic lifetime as an extra time scale. Details of the model used for the fitting are given in Ref. 18 .

Systematic cross-correlation data analysis allowed us to study the SD rate as a function of different parameters. We have plotted in Fig. 4(a) the diffusion rate $\gamma_{d}$ of the CX line as a function of pumping power at two different temperatures. It can be seen that the diffusion rate increases as the pumping power is raised. Higher temperatures lead also to larger diffusion rates.

To explain the power and temperature dependency shown in Fig. 4, we have in mind a model with charge traps in the vicinity of the QD. ${ }^{28}$ Electrical charges are randomly trapped and induce a fluctuating electric field, leading to spectral diffusion of QD transitions via the Stark effect. To account for the observed SD broadened linewidth, charge traps need to be located within a few nanometers of the QD. ${ }^{4,8}$ In the case of $\mathrm{ZnSe}$ the residual doping is of the $n$ type, and we assume that the charges are electrons. Surprisingly, we have observed that neither the SD-induced linewidth (i.e., amplitude of the spectral fluctuations) nor the line shape depend on the pumping power, as shown in Fig. 4(b). A possible explanation would be that there is only room for a single charge exploring several trapping sites around the QD and that additional charges are

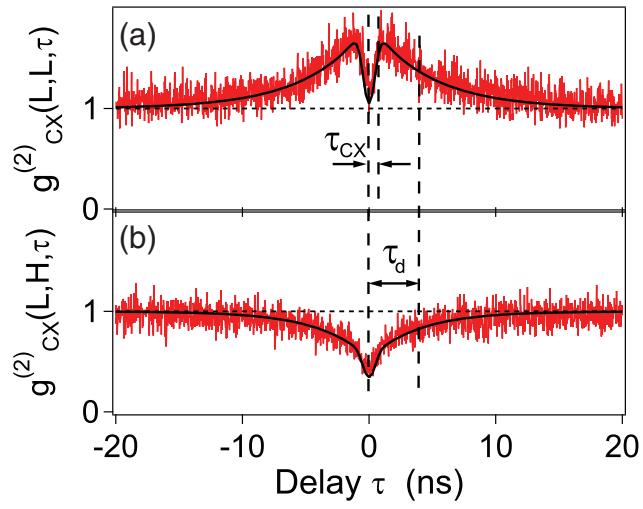

FIG. 2. (Color online) Spectral diffusion of the charged exciton (CX) line (see text). (a) Autocorrelation of one half of the profile showing the bunching due to $\mathrm{SD}\left(\tau_{d}=4 \mathrm{~ns}\right)$ and the narrower singlephoton antibunching. (b) Cross correlation between the two halves of the profile displaying the antibunching due to SD with the same characteristic time $\tau_{d}=4 \mathrm{~ns}$. These two plots have been obtained on the same QD with the same excitation power. The solid lines are fits with the model explained in Ref. 18.

blocked by Coulomb repulsion. Indeed, the presence of several charges, which could be induced by large pump power, would lead to a broadening of the SD-induced linewidth, which is not observed.

The charge number $c$ trapped around the QD is governed by the following rate equation:

$$
\frac{d c}{d t}=\gamma_{\text {in }}^{\prime}(T)\left(N+N_{o}\right)(1-c)-\gamma_{\text {out }}^{\prime}(T) N c,
$$

where the first (second) term is the loading (escape) term. The loading term is proportional to $(1-c)$ to account for the fact that the maximum number of charge is 1 . The loading rate $\gamma_{\text {in }}^{\prime}(T)\left(N+N_{o}\right)$ is proportional to the number of electrons $N+N_{o}$ in the ZnSe barrier, where $N$ corresponds to the number of photocreated electron-hole pairs in the barrier and $N_{o}$ corresponds to the residual doping. For the escape mechanism, we assume that the prominent effect is the recombination of the trapped electron with a photocreated hole. This leads to an escape rate $\gamma_{\text {out }}^{\prime}(T) N$ that is also proportional to $N$. We assume an activation type behavior $\sim \exp \left(-E_{a} / k T\right)$ for the temperature dependency of $\gamma_{\text {in }}^{\prime}(T)$ and $\gamma_{\text {out }}^{\prime}(T){ }^{29}$ The energy $E_{a}$ corresponds to shallow potential fluctuations experienced by the charges in the barrier. Raising the temperature increases their diffusion length and makes it more likely for the electrons (holes) to load (empty) the deeper traps, causing the spectral diffusion of the QD.
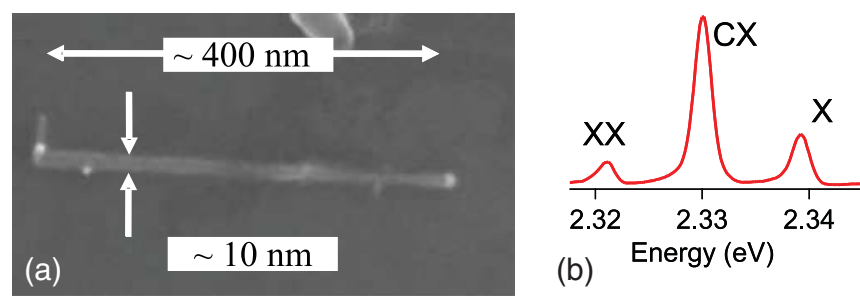

FIG. 3. (Color online) (a) Scanning electron microscope image of a $\mathrm{ZnSe}$ nanowire containing a CdSe quantum dot. (b) Photoluminescence spectrum. 


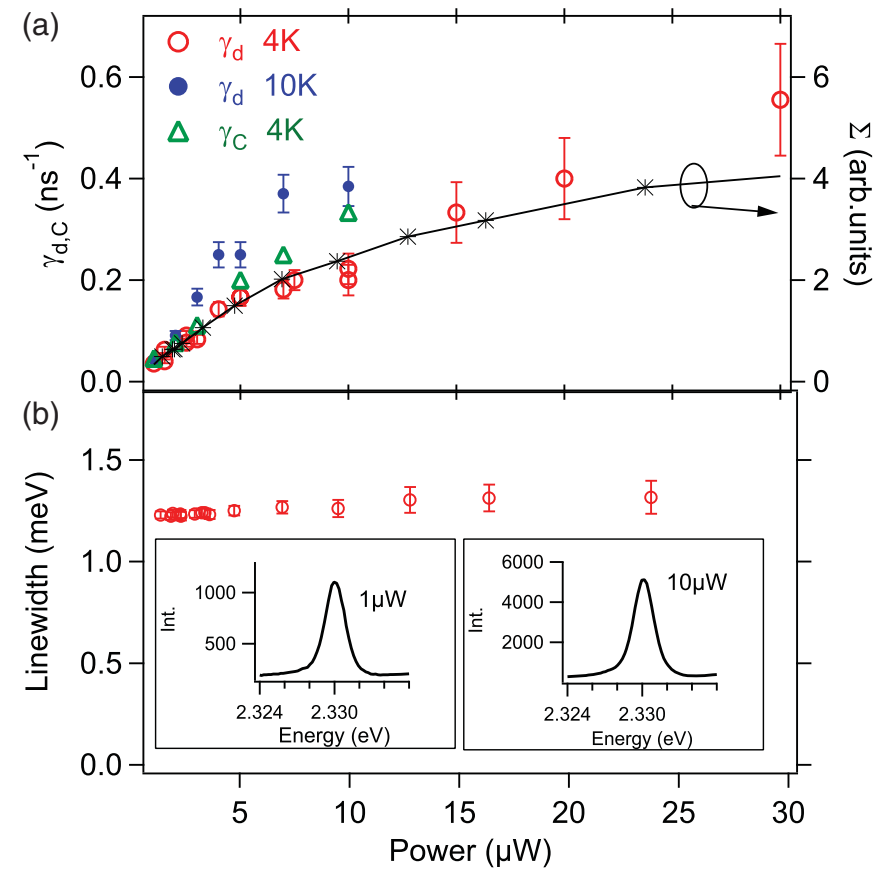

FIG. 4. (Color online) (a) Left axis: Diffusion rate $\gamma_{d}$ at $T=4 \mathrm{~K}$ (open red circles) and $T=10 \mathrm{~K}$ (solid blue circles) and the charged to neutral hopping rate $\gamma_{C}$ (open green triangles; see text) as a function of exciting power. Right axis: The black stars are proportional to the total amount of light $\Sigma$ emitted by X, CX, and XX and a fair approximation of the total number of carrier $N$ in the NW. The saturation pumping power for the charged exciton is $4 \mu W$. (b) Corresponding linewidth as a function of exciting power at $T=4 \mathrm{~K}$. The insets are examples of two spectra taken at different powers, showing that not only the linewidth but also the shape is unchanged.

From the increase of spectral diffusion with temperature, $\gamma_{d}(10 K) / \gamma_{d}(4 K)=1.85 \pm 0.15$ at low powers, we can infer an activation energy $E_{a}=0.35 \pm 0.05 \mathrm{meV}$.

Within this model, the spectral diffusion rate $\gamma_{d}$ is given by

$$
\gamma_{d}=\gamma_{\text {in }}^{\prime}(T) N_{o}+\left[\gamma_{\text {in }}^{\prime}(T)+\gamma_{\text {out }}^{\prime}(T)\right] N .
$$

From the experimental data displayed in Fig. 4, it appears that the diffusion rate approaches zero as the pumping power vanishes. This means that the contribution of $\gamma_{\text {in }}^{\prime}(T) N_{o}$ is negligible compared to the term depending on the carrier number $N$. As seen in Fig. 4(a) the diffusion rates at $T=4 \mathrm{~K}$ and $T=10 \mathrm{~K}$ exhibit a sublinear power dependency. So does the total light intensity $\Sigma=\mathrm{X}+\mathrm{XX}+\mathrm{CX}$ emitted within the exciton, biexciton, and charged exciton lines. The quantity $\Sigma$ is proportional to the carrier number $N$ in the nanowire as long as these three lines are not saturated, which is the case up to a pump power of $4 \mu \mathrm{W}$ in our situation. For larger pump power, higher-order multiexcitonic lines appear away from the detected spectral window, and the $\Sigma$ power dependency slightly underestimates the total amount of carrier in the nanowire. The similar power dependencies of $\gamma_{d}$ and $\Sigma$ are therefore a good indication of the validity of equation (2), but they are not unambiguous proof. Additionally, we mention that the sublinear power behavior of the total amount of the carrier could also partly be attributed to Auger scattering in the barrier. ${ }^{28,30}$

We compare now the spectral diffusion rate with the charged to neutral hopping rate $\gamma_{C}$, which is also displayed in Fig. 4. This rate has been extracted from the width of the bunching peak of the autocorrelation of the whole line of the charged exciton. $^{23}$ The similar hopping rate $\gamma_{C}$ power dependency suggests a charged-neutral hopping mechanism similar to the spectral diffusion one, as exposed above. ${ }^{31}$ The comparison between the absolute quantitative values of $\gamma_{C}$ and $\gamma_{d}$ is not fully reliable since the data were acquired on different days. Nevertheless, the close values of $\gamma_{C}$ and $\gamma_{d}$ are in favor of a scenario in which a spectral diffusion event is a jump into the other charge state and back with a modified surrounding charge distribution.

To summarize, we have used a recently developed method ${ }^{18}$ to measure spectral diffusion of single emitters with a subnanosecond resolution. We have used this technique to study, as a function of pumping power and temperature, the spectral diffusion characteristics of the photoluminescence of a single $\mathrm{CdSe}$ quantum dot inserted in a $\mathrm{ZnSe}$ nanowire. We have found a characteristic SD time $\tau_{d}$ of a few nanoseconds and have given good indications that this rate $\gamma_{d}=1 / \tau_{d}$ is proportional to the carrier number in the wire. In addition to the power-independent linewidth, these findings support a model where a single charge is wandering within a few nanometers around the quantum dot, suggesting a spectral diffusion mechanism closely related to the charged-neutral QD hopping events.

T.A. acknowledges support from Deutscher Akademischer Austauschdienst (DAAD). Part of this work was supported by the European project QAP (Contract No. 15848).
${ }^{1}$ W. P. Ambrose and W. E. Moerner, Nature (London) 349, 225 (1991).

${ }^{2}$ T. Plakhotnik and D. Walser, Phys. Rev. Lett. 80, 4064 (1998).

${ }^{3}$ S. A. Empedocles, D. J. Norris, and M. G. Bawendi, Phys. Rev. Lett. 77, 3873 (1996).

${ }^{4}$ H. D. Robinson and B. B. Goldberg, Phys. Rev. B 61, R5086 (2000).

${ }^{5}$ J. Seufert, R. Weigand, G. Bacher, T. Kümmell, A. Forchel,

K. Leonardi, and D. Hommel, Appl. Phys. Lett. 76, 1872 (2000).

${ }^{6}$ V. Türck, S. Rodt, O. Stier, R. Heitz, R. Engelhardt, U. W. Pohl, D. Bimberg, and R. Steingruber, Phys. Rev. B 61, 9944 (2000).
${ }^{7}$ L. Besombes, K. Kheng, L. Marsal, and H. Mariette, Phys. Rev. B 65, 121314 (2002).

${ }^{8}$ S. A. Empedocles and M. G. Bawendi, Science 278, 2114 (1997).

${ }^{9}$ L. Besombes, Y. Leger, J. Bernos, H. Boukari, H. Mariette, J. P. Poizat, T. Clement, J. Fernández-Rossier, and R. Aguado, Phys. Rev. B 78, 125324 (2008).

${ }^{10}$ C. Simon et al., Eur. Phys. J. D 58, 1 (2010).

${ }^{11}$ C. Simon, Y.-M. Niquet, X. Caillet, J. Eymery, J.-P. Poizat, and J.-M. Gérard, Phys. Rev. B 75, 081302 (2007). 
${ }^{12}$ A. Auffèves-Garnier, C. Simon, J.-M. Gérard, and J. P. Poizat, Phys. Rev. A 75, 053823 (2007).

${ }^{13}$ J. L. O’Brien, A. Furusawa, and J. Vučković, Nat. Photon. 3, 687 (2009).

${ }^{14}$ A. Beveratos, R. Brouri, T. Gacoin, A. Villing, J. P. Poizat, and P. Grangier, Phys. Rev. Lett. 89, 187901 (2002).

${ }^{15}$ E. Waks, K. Inoue, C. Santori, D. Fattal, J. Vuckovic, G. S. Solomon, and Y. Yamamoto, Nature (London) 420, 762 (2002).

${ }^{16}$ E. Knill, R. Laflamme, and G. J. Milburn, Nature (London) 409, 46 (2001); see also A. Kiraz, M. Atatüre, and A. Imamoğlu, Phys. Rev. A 69, 032305 (2004) ; P. Kok, W. J. Munro, K. Nemoto, T. C. Ralph, J. P. Dowling, and G. J. Milburn, Rev. Mod. Phys. 79, 135 (2007).

${ }^{17}$ P. Palinginis, S. Tavenner, M. Lonergan, and H. Wang, Phys. Rev. B 67, 201307 (2003).

${ }^{18}$ G. Sallen, A. Tribu, T. Aichele, R. André, L. Besombes, C. Bougerol, M. Richard, S. Tatarenko, K. Kheng, and J. Ph. Poizat, Nat. Photon. 4, 696 (2010).

${ }^{19}$ A. Zumbusch, L. Fleury, R. Brown, J. Bernard, and M. Orrit, Phys. Rev. Lett. 70, 3584 (1993).

${ }^{20}$ L. Coolen, X. Brokmann, P. Spinicelli, and J.-P. Hermier, Phys. Rev. Lett. 100, 027403 (2008).
${ }^{21}$ L. F. Marshall, J. Cui, X. Brokmann, and M. G. Bawendi, Phys. Rev. Lett. 105, 053005 (2010).

${ }^{22}$ T. Aichele, A. Tribu, C. Bougerol, K. Kheng, R. André, and S. Tatarenko, Appl. Phys. Lett. 93, 143106 (2008).

${ }^{23}$ G. Sallen, A. Tribu, T. Aichele, R. André, L. Besombes, C. Bougerol, S. Tatarenko, K. Kheng, and J. Ph. Poizat, Phys. Rev. B 80, 085310 (2009).

${ }^{24}$ A. Tribu, G. Sallen, T. Aichele, R. André, J.-Ph. Poizat, C. Bougerol, S. Tatarenko, and K. Kheng, Nano Lett. 8, 4326 (2008).

${ }^{25}$ Y. Li, F. Qian, J. Xiang, and C. M. Lieber, Mater. Today 9, 18 (2006).

${ }^{26}$ V. Türck, S. Rodt, R. Heitz, O. Stier, M. Strassburg, U. W. Pohl, and D. Bimberg, Phys. Status Solidi B 224, 217 (2001).

${ }^{27}$ B. Patton, W. Langbein, and U. Woggon, Phys. Rev. B 68, 125316 (2003).

${ }^{28}$ A. Berthelot, I. Favero, G. Cassabois, C. Voisin, C. Delalande, $\mathrm{Ph}$. Roussignol, R. Ferreira, and J. M. Gérard, Nat. Physics 2, 759 (2006).

${ }^{29}$ H. Kamada and T. Kutsuwa, Phys. Rev. B 78, 155324 (2008).

${ }^{30}$ K. E. O’Hara, J. R. Gullingsrud, and J. P. Wolfe, Phys. Rev. B 60, 10872 (1999).

${ }^{31}$ M. H. Baier, A. Malko, E. Pelucchi, D. Y. Oberli, and E. Kapon, Phys. Rev. B 73, 205321 (2006). 